\title{
Flujo de información destino-cliente a través de alojamientos privados. Municipio Plaza de la Revolución.
}

\author{
Flow of customer-destination information through private \\ accommodations. Plaza de la Revolución
}
Claudia Castellanos Cartaya. ${ }^{1}$, Daikel Adriano Abreu Rodríguez. ${ }^{2}$, Dailén Abreu Rodríguez. ${ }^{3} \&$ Arnaldo Rojas López. ${ }^{4}$

\begin{abstract}
The information in the tourist activity it is of vital importance. It is used for the operation and the management of the companies, destinations and organization and execution of the trip of the clients. The present research is carried out about the information to the visitors at the Plaza de la Revolución municipality, Havana. It includes the analysis of private accommodations as tourist information mediators. For this purpose, the bibliographical revision, the interview, the analysis of documents and the observation as methods and techniques for collecting information were used. A diagnosis of the current situation of this phenomenon was done, in which the existence of insufficiencies in the transmission of information to the clients staying in private accommodations was corroborated. That is why a proposal of a destination-client information flow mechanism was made, using the private accommodation managers as information mediators.
\end{abstract}

Key words: private accommodation, information mediators, information flow.

\footnotetext{
${ }^{1}$ Trabajadora por cuenta propia, claudia.cascar@nauta.cu

2 Universidad de la Habana, Ministerio de Educación Superior, daikel_abreu@ftur.uh.cu, ORCID:0000-0001-8592-4357

${ }^{3}$ Empresa Extrahotelera Palmares, Ministerio del Turismo, dailenar@nauta.cu

4 Trabajador por cuenta propia, rojaslopezarnaldo@gmail.com
} 


\section{Resumen}

En la actividad turística es de vital importancia la información, es utilizada para la operación y la gestión de las empresas, destinos y la organización y ejecución de los viajes de los clientes. El presente es una investigación realizada sobre el tema de la información a los visitantes en el municipio Plaza de la Revolución, La Habana. Comprende el análisis de los alojamientos privados como infomediarios turísticos. Para ello se empleó la revisión bibliográfica, la entrevista, el análisis de documentos y la observación como métodos y técnicas de recogida de información. Se presentó un diagnóstico de la situación actual de dicho fenómeno, donde se corroboró la existencia de insuficiencias en la transmisión de información a los clientes que se hospedan en casas particulares, lo que condujo a la propuesta de un mecanismo de flujo de información destino-cliente usando como infomediarios a los gestores de alojamientos privados.

Palabras claves: alojamientos privados, infomediarios, flujo de información.

\section{Introducción}

En la gestión de los Destinos Turísticos (DT) es de vital importancia el intercambio de información constante a todos los agentes que participan en el mismo. Esta juega un papel decisivo para asumir los nuevos retos, los cambios tecnológicos, evaluar la importancia de la actividad en la economía y sociedad, y lograr un óptimo aprovechamiento de los recursos materiales.

La información, constituye un instrumento esencial de apoyo para las empresas turísticas y permite a los visitantes reales o potenciales conocer mejor los servicios y los productos existentes para tomar de manera más acertada su decisión de compra.

La Oficina Nacional de Información Turística en Cuba es una entidad encargada de recopilar información para la gestión turística e informar a los visitantes una vez en el destino. En el caso particular de La Habana desde 1998 existe la Red de Centros de Información al Turista (INFOTUR), situados en lugares de alta concentración de turistas y subordinados a esta. (Oficina Nacional de Información Turística, 2013a). Según Valdés (2011) desde el 2005-2008, del total de turistas que visitaron La Habana, un 8\% asistieron a los INFOTUR en busca de información.

Según Ramón Martín en entrevista realizada en noviembre del 2013, la mayor parte de la información que reciben los turistas durante su estancia en el destino es en los alojamientos, por lo que los mismos juegan un importante papel como infomediarios.

El arrendamiento de viviendas y habitaciones de cuentapropistas ha ido en ascenso debido al incremento en cantidad de alojamientos destinados a este fin y de turistas que optan por este. (Cubavisión, 2014a). En el período 2008-2013, las habitaciones de propiedad privadas crecieron en un $76 \%$. En ese mismo período los turistas que se hospedaron en casas particulares, alcanzaron el $20 \%$ del total de llegadas de visitantes al país. (Pérez, 
2014). No obstante, sus gestores no poseen toda la información necesaria para su satisfacción.

Teniendo en cuenta los diferentes definiciones encontradas en la literatura los autores consideraron al Destino Turístico como un espacio geográficamente delimitado que posee recursos y condiciones para el desarrollo del turismo, el cual se gestiona y comercializa teniendo en cuenta su carácter integral y donde tiene lugar una realidad sistémica compleja integrada no solo por elementos del sistema turístico y elementos del sistema territorial que pueden ser espaciales, administrativos y productivos, sino también por las relaciones que entre ellos se establecen y que en su dinámica generan una imagen percibida por los visitantes, los que son atraídos a dicho destino mediante productos adaptados a sus satisfacciones buscadas.

Guevara y colaboradores (2011) plantean que un Sistema de Gestión de Destino tiene dos elementos básicos: la integración de toda la información relevante en un sistema automatizado de información y la recolección de información estadística que permita cuantificar las relaciones entre los usuarios de los servicios y el destino en sus diversas dimensiones.

$\mathrm{Al}$ ser el turismo fuente y origen de un gran volumen de datos surge la necesidad de un Sistema de Información Turística (SIT)que integre cantidad y calidad de información, cuestión central para los distintos agentes que participan en el mismo. En el ámbito de un destino turístico, el SIT, es "proceso permanente y sistematizado de recopilación, tratamiento, ordenación y distribución de la información precisa para los objetivos de planificación, de acción y de evaluación turística para los distintos agentes turísticos públicos y empresariales de un destino“. (Centeno, 2011:14)

Los SIT se dividen en dos tipos: los Sistemas de Información al Visitante (SIV)y los Sistemas de Información para la Gestión del Destino (SIGD).

Castro (2013) plantea que dentro de las fuentes de información más utilizadas por los SIGD se encuentran: los propios visitantes, las estadísticas vinculadas a los niveles de gestión de las entidades turísticas, las encuestas a los oferentes, las propias empresas proveedoras de servicios para el turista y las oficinas que se encargan de aglutinar o recopilar información.

Los consumidores de turismo, cada vez buscan productos turísticos más personalizados y servicios inteligentes que les proporcionen información de alta calidad, en cualquier momento y en cualquier lugar y habitualmente en tiempo real. Los distintos Destinos Turísticos se enfrentan al desafío de garantizar esas ofertas de forma moderna, interactiva, accesible y fiable, en ello se basa su competitividad. (Rodríguez y Caballero, 2012). 
Los visitantes reales y potenciales se han visto beneficiados por el desarrollo tecnológico de la información y las comunicaciones en varios aspectos. Los principales son: Internet y Web 2.0, televisión y otros medios públicos de información y telefonía móvil. (Martín 2014:148)

Las informaciones que obtienen los turistas antes de realizar el viaje son suministradas por: turoperadores extranjeros mediante agencias de viajes minoristas extranjeras que brindan atención personalizada o mediante los sitios web en Internet; por entidades extranjeras en diferentes puntos de ventas mediante libros, mapas y guías turísticas; agencias receptivas nacionales a través de sus sitios web en Internet; Oficina Nacional de Información Turística a través de sus sitios web propios en Internet, materiales impresos, anuncios televisivos, vallas promocionales, redes sociales, opiniones de amigos y familiares entre otros. (Martín y Abreu, 2013).

Mientras que la información que reciben los turistas durante su estancia en el destino son suministradas en su gran mayoría por: las agencias receptivas nacionales a través de los Buroes o puntos de ventas donde se les brida información directa personal; la Oficina Nacional de Información Turística a través de sus sitios web propios en Internet; las empresas estatales del destino ya sean alojamientos, restaurantes, centros nocturnos y otras entidades de ocio; los negocios privados tanto de alojamiento como de restauración; INFOTUR mediante información directa personal, libros, mapas y guías turísticas. (Martín y Abreu, 2013).

La Oficina Nacional de Información Turística tiene como misión proveer la información veraz y oportuna que demandan los visitantes y el turismo doméstico, a través de la Red Nacional de los Centros de Información y Sitios web online. (Oficina Nacional de Información Turística, 2013b).

A partir de los elementos abordados se identificaron y definieron como componentes del diseño de la investigación los siguientes:

Teniendo en cuenta insuficiencias en la información que se brinda a los clientes durante su estancia en alojamientos privados el objetivo de la investigación fue diseñar un mecanismo de flujo de información entre la Oficina Nacional de Información Turística y el cliente en el destino a través de los alojamientos privados.

\section{Método}

En el estudio realizado se utilizaron métodos empíricos como la entrevista ya observación. Los métodos teóricos utilizados fueron: el analítico - sintético, el histórico - lógico y el inductivo - deductivo. 


\section{Procedimiento utilizado para el desarrollo de la investigación}

La investigación se desarrolló a través de las siguientes etapas: planteamiento y justificación del problema, construcción del modelo teórico, determinación de la población objeto de estudio y la muestra correspondiente, elaboración de los métodos y técnicas para la recopilación de información, aplicación de los métodos y técnicas, procesamiento y análisis de la información, y elaboración del informe. (Sancho y colaboradores, 2001).

Se decidió realizar el estudio en el municipio Plaza de la Revolución debido a que el mismo cuenta con la mayor cantidad de TCP de alojamiento de provincia Habana. Las unidades de estudio fueron:

- Los trabajadores de las entidades de alojamiento privado en Plaza de la Revolución.

- Especialistas en el tema

- Estudiantes de segundo año de la Licenciatura en Turismo que se encontraban realizando sus prácticas docentes en el INFOTUR de Plaza de La Revolución.

Las entrevistas realizadas a 40 trabajadores de los alojamientos privados en el municipio Plaza de la Revolución en los meses de enero y febrero del 2014, tuvieron como finalidad conocer cómo funciona el flujo de información gestor-cliente. Específicamente se indagó por la información que solicitan los turistas y por tanto aquellas que necesitan tener a su disposición estos TCP para poder brindárselas a sus arrendados, los momentos en que solicitan las informaciones y las formas en que desean recibirlas.

Las entrevistas a los especialistas se aplicaron entre diciembre del 2013 y marzo del 2014 con la finalidad de diagnosticar la situación actual existente en el Sistema de Información al Visitante en el país y de forma más específica en Plaza de la Revolución, conocer las normativas legales por las que se rige el TCP e indagar acerca del trabajo que ha realizado la delegación del MINTUR en La Habana respecto a los negocios privados. Estas se realizaron hasta que fue necesario profundizar en alguna información sobre el tema en cuestión utilizándose el criterio de completamiento de la información.

Las entrevistas a los estudiantes de segundo año de la Facultad de Turismo del curso 2013-2014 se efectuaron para conocer el trabajo que han ido llevando a cabo en colaboración con INFOTUR en Plaza de la Revolución.

Una vez finalizado el trabajo de campo se procesó la información cualitativa obtenida, se elaboró la propuesta del mecanismo de flujo de información teniendo en cuenta los resultados obtenidos y después de realizado un análisis del funcionamiento de INFOTUR 
en el municipio Plaza de la Revolución, así como de las proyecciones de la Oficina Nacional de Información Turística.

Un total de 29 mil 952 trabajadores privados en todo el país desarrollan la actividad de arrendamiento de habitaciones. (Cubavisión, 2014a).

Abreu (2011) plantea que en la actualidad algunos negocios privados forman parte del Sistema Turístico Cubano como por ejemplo los alojamientos privados que brindan sus servicios a extranjeros, paladares y otros. Al cierre de febrero del año 2014 la cifra total de trabajadores por cuenta propia de alojamiento en La Habana era de 10576. (Cubavisión, 2014b).

A decir de Pérez (2014) durante el 2008 y el 2013 los turistas que eligieron este tipo de establecimiento alcanzaron el $20 \%$ del total de llegadas de visitantes al país. En particular, en esos últimos tres años, creció en un $2 \%$ anual, alcanzando el $23 \%$ de hospedados en la Isla.

En el 2012 y el 2013 los visitantes alojados en casas particulares continuaron situándose entre la quinta y la cuarta parte del total de visitantes que arribaron al país. (Abreu y Martín, 2013) Pérez (2014) plantea que Plaza de la Revolución es el municipio capitalino que cuenta con la cifra más elevada TCP de alojamiento en el presente año contando con el 39 por ciento de toda la provincia y el 13 por ciento del territorio nacional.

\section{Resultados y Discusión}

Flujo de información: gestor de alojamiento privado- cliente, en el municipio Plaza de la Revolución.

De los trabajadores privados entrevistados, su totalidad planteó que los turistas que se hospedan en sus casas solicitan información durante toda su estancia y que las mismas varían en cuanto al volumen y tipo de información y teniendo en cuenta si es la primera vez que visitan Cuba, los países de procedencia, edades y sexo de los clientes; pero que en cualquiera de los casos el requerimiento es muy frecuente y de variada índole. Las informaciones solicitadas por los clientes según temáticas y las fuentes por las que los TCP obtenían las informaciones que les brindaban a sus clientes se reflejan en las gráficas siguientes. 

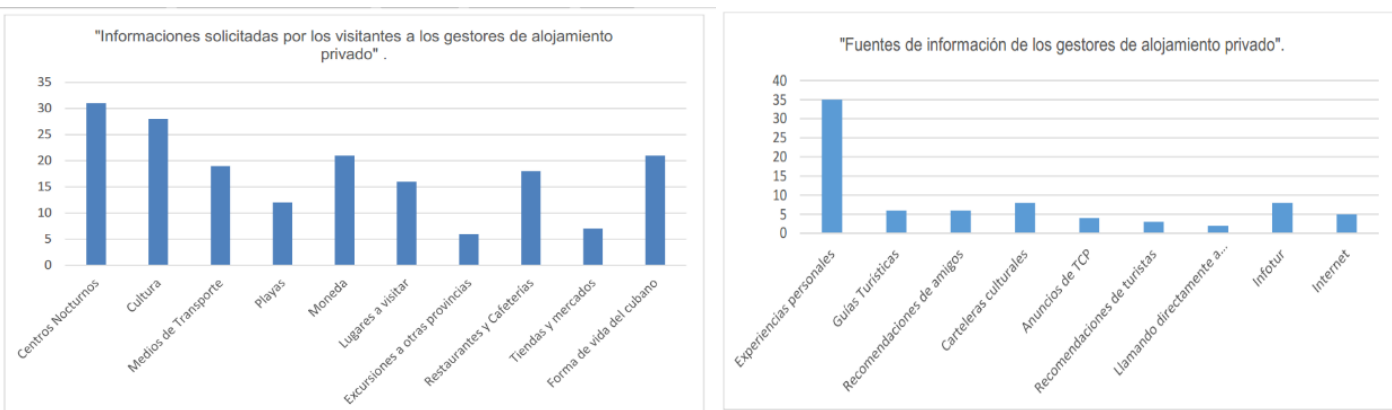

Fuente de elaboración propia a partir de las entrevistas realizadas

Según los TCP entrevistados los visitantes se preocupan en mayor medida por centros nocturnos y la cultura. La mayor parte de ellos expresaron que las informaciones que brindaban eran basadas en su experiencia personal.

Es significativo el hecho de que todos los entrevistados expresaron que les gustaría y sería de gran utilidad recibir información actualizada del país, expresando más de la mitad (24) de ellos, que no siempre tenían a su alcance las informaciones necesarias para satisfacer todas las interrogantes de sus clientes. Esto evidencia la existencia de vacíos informativos en dichos negocios lo que pudiera repercutir de forma negativa en la satisfacción de los visitantes durante su estancia, así como una disminución de los ingresos turísticos por la realización de actividades complementarias.

Al indagar en qué se basaban los gestores para dar sus recomendaciones y cuáles eran los lugares que más recomendaban a los turistas las respuestas fueron las siguientes:
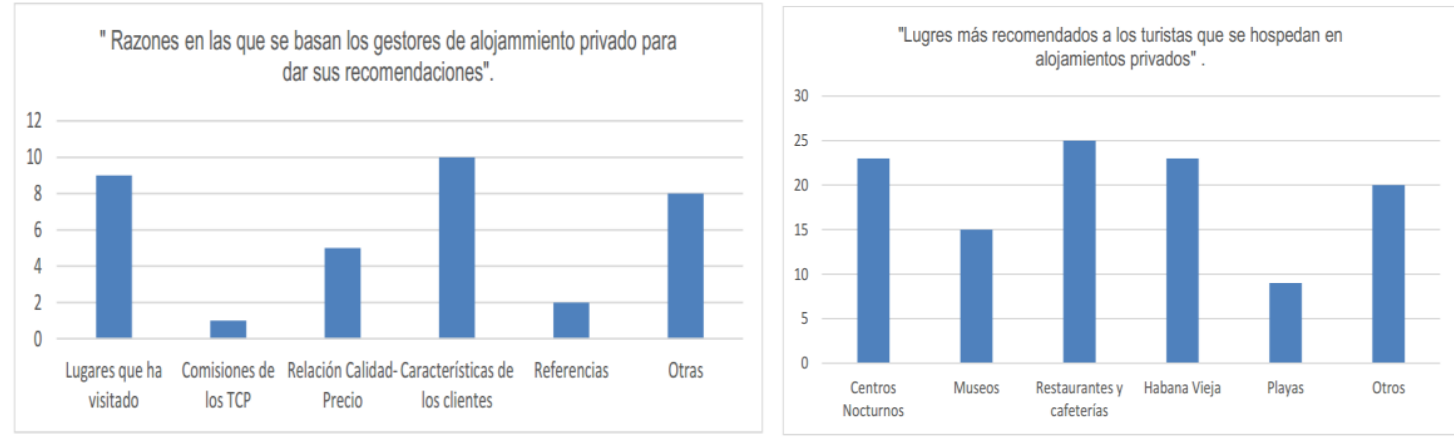

Fuente de elaboración propia a partir de las entrevistas realizadas

Sobre las características sociodemográficas de los turistas que visitan los alojamientos privados, los gestores de estos negocios no ofrecieron respuestas concretas, lo cual puede deberse al desinterés o recelo hacia ese tipo de información, puesto que los mismos tienen la obligación de llevar un registro con los datos de sus clientes.

Al indagar por la cantidad de gestores que recomendaban visitar INFOTUR a sus clientes se obtuvo que existe un escaso aprovechamiento de los servicios que brinda INFOTUR. 
Al preguntar sobre si dichos TCP conocía la posibilidad que brinda INFOTUR para promocionarse en la Guía de Alojamiento del Destino solamente 8 de los entrevistados expresaron que la conocían, pero no a profundidad, denotando esto la necesidad de aumentar la divulgación de dicha oferta.

Los gestores de alojamiento expresaron que utilizaban diferentes vías para promocionarse: páginas web, recomendaciones, carteles a la entrada del alojamiento, redes sociales, tarjetas de presentación, aplicaciones para móviles androides y computadoras, páginas amarillas de la Guía Telefónica de Etecsa y Trípticos

\section{Propuesta del Mecanismo de Flujo de Información entre la Oficina Nacional de Información Turística y los clientes en el destino a través de los negocios privados de alojamiento.}

Después de haber realizado un análisis del funcionamiento de INFOTUR en el municipio Plaza de la Revolución, así como de las proyecciones de la Oficina Nacional de Información Turística y teniendo en cuenta las entrevistas llevadas a cabo durante la investigación, tanto a los arrendadores como a los especialistas, se detectaron las siguientes insuficiencias:

- La Oficina Nacional de Información Turística no contempla a los alojamientos privados como integrantes de los subsistemas Tributario y Diseminador.

- Los gestores de alojamientos no siempre tienen a su alcance las informaciones necesarias para satisfacer todas las interrogantes de sus clientes.

- El mecanismo que se propone persigue el mejoramiento del Sistema de Información al Visitante en el destino permitiendo:

- Satisfacer las demandas informativas de los visitantes que optan por la modalidad de alojamiento privado en nuestro país, propiciando la optimización en la utilización de los recursos culturales, naturales y económicos.

- Influir en una mayor satisfacción y calidad de la estancia del visitante hospedado en alojamientos privados.

- Propiciar un mayor gasto per cápita de los turistas alojados en casas particulares durante su estancia.

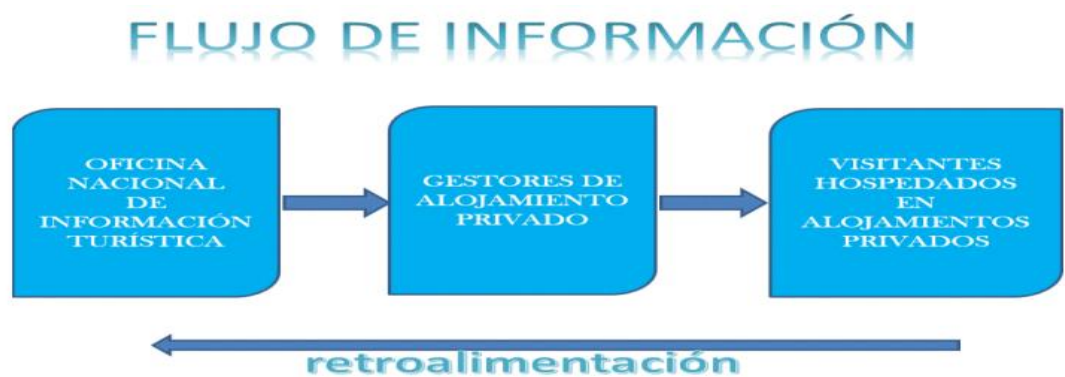

Fuente: elaboración propia. 
Se propuso implementar este mecanismo de flujo de información a partir de enero del 2015 en el municipio Plaza de la Revolución para su posteriormente aplicarlo en el resto del país. Para ello se hace necesario tener en cuenta los actores, las informaciones que recibirán y brindarán, así como las vías necesarias para este propósito.

1. Los principales actores como se reconoce en la gráfica propuesta son: Oficina Nacional de Información Turística, gestores de alojamientos privados, visitantes del destino que se hospedan en casas particulares.

2. Información: esta deberá cumplir con los siguientes requisitos: calidad, oportunidad, cantidad y relevancia.

3. Se brindarán informaciones del destino de forma general, priorizando aquellas que solicitan con mayor frecuencia los turistas a sus caseros, referidas anteriormente en la primera gráfica.

4. Todas estas informaciones se recogerán en formatos impresos y digitales. Dentro de los materiales impresos se encuentran: guía del destino, guías temáticas, mini guías, directorio turístico de Cuba, tabloide, sueltos, multimedias y videos y aplicaciones.

5. Las utilizaciones de las aplicaciones resultan beneficiosas ya que el país no tendrá que dedicar grandes inversiones al desarrollo y distribución de materiales impresos. La principal ventaja de los mismos radica en que los usuarios tendrán la posibilidad de trabajar en cualquier PC o móvil sin necesidad de conexión a ninguna red.

6. Vía por las que se distribuirán las informaciones: los INFOTUR, correo postal, correo electrónico, talleres de corta duración.

Es válido aclarar que además de las formas propuestas se continuará brindando información a los TCP y visitantes del destino por las vías tradicionales: teléfono y atención directa en los INFOTUR.

\section{Conclusiones}

- El turismo necesita de los Sistemas de Información para integrar cantidad y calidad de información, cuestión central para los distintos agentes que participan en el mismo (sector público y privado, los turistas y comunidad receptora).

- Las entrevistas realizadas a los gestores de alojamiento privado arrojaron que la totalidad de los visitantes que se hospedan en sus casas solicitan información durante su estancia para lo cual tienen limitaciones por no tener a su alcance las informaciones necesarias.

- La implementación del mecanismo de flujo de información propuesto entre la Oficina Nacional de Información Turística y los clientes en el destino a través de los alojamientos privados contribuirá al mejoramiento del Sistema de Información al Visitante. 


\section{Bibliografía}

Abreu Rodríguez, D. Turismo, Sector Privado y Capital Humano. Trabajo final. Curso de Sociología del Trabajo. Sancti Spíritus. Cuba. 2011.

Castro Rodríguez, P. Situación actual y perspectivas de la información a los turistas. Trabajo de Diploma en Opción al Título de Licenciado en Turismo, Facultad de Turismo, Universidad de La Habana. La Habana. Cuba. 2013.

Centeno, H.; Doffourt, G.; García, N.; González, E.; Gómez, G.; Granado, L.; Loyo, S.; Pérez, A. y Pérez, D. Sistemas de Información Turísticos. 2011.

Cubavisión. Mesa Redonda Informativa: "Situación actual del trabajo por cuenta propia”. 2da parte. Transmitido el 20 de marzo de 2014.

Cubavisión. Mesa Redonda Informativa. "Situación actual del trabajo por cuenta propia". 1ra parte. Transmitido el 19 de marzo de 2014.

Guevara, A.; Caro, J.L.; Aguayo, A.; Rossi, C. y Leiva, J.L. Sistema Integrado de Gestión de Destinos. VIII Congreso "Turismo y Tecnologías de la Información y las Comunicaciones" Turitec. (2010).

Martín Fernández, R. Principios de Organización y Práctica de Turismo. Segunda Parte. Texto Docente. La Habana. Cuba 2014. Editorial Félix Varela.

Martín Fernández, R. y Abreu Rodríguez, D.A. Flujo de Información Turística. Documento de Trabajo del Centro de Estudios Turísticos de la Facultad de Turismo. 2013.

Oficina Nacional de Información Turística. Características del Infotur de Plaza de la Revolución. Documento de trabajo. La Habana. Cuba. (2013a).

Oficina Nacional de Información Turística. Manual de Gestión de la Comunicación. Documento de trabajo. La Habana. Cuba. (2013b).

Pérez Martínez, Y. “Administración jurídica y turismo no estatal”. Tesis en Opción al Título de Doctor en Ciencias, Facultad de Derecho, Universidad de La Habana. La Habana. Cuba. 2014. (Predefensa)

Rodríguez Díaz, B. y Caballero Fernández, R. Sistema de ayuda al turista. Modelo para la planificación de un viaje personalizado. Estudios y perspectivas en turismo. Universidad de Málaga. España. (2012). ISSN 1851-1732.

Sancho Pérez, A.; Cabrer Borrás, B.; García Mesanat, G.; Pérez Mira, J.M.; García Pedro, P. y García Sanchis, M. Apuntes de Metodología de la Investigación en Turismo. Madrid. España. 2001.

Valdés, L; Martín, R,: Aza, R,: Del Valle, E. y Delgado, A. La Demanda Turística hacia La Habana. Implementación adaptada del Sistema de Información Turística de Asturias. Asturias, España. 2011.

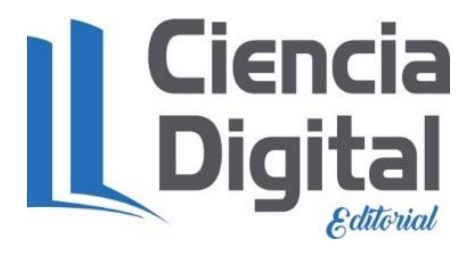




\section{PARA CITAR EL ARTÍCULO INDEXADO.}

Castellanos Cartaya, C., Abreu Rodríguez, D. A., Abreu Rodríguez, D., \& Rojas López, A. (2021). Flujo de información destino-cliente a través de alojamientos privados. Municipio Plaza de la Revolución. Explorador Digital, 5(2), 23-33. https://doi.org/10.33262/exploradordigital.v5i2.1650

\section{$\lfloor$ Ciencia}

El artículo que se publica es de exclusiva responsabilidad de los autores y no necesariamente reflejan el pensamiento de la Revista Explorador Digital.

El artículo queda en propiedad de la revista y, por tanto, su publicación parcial y/o total en otro medio tiene que ser autorizado por el director de la Revista Explorador Digital.
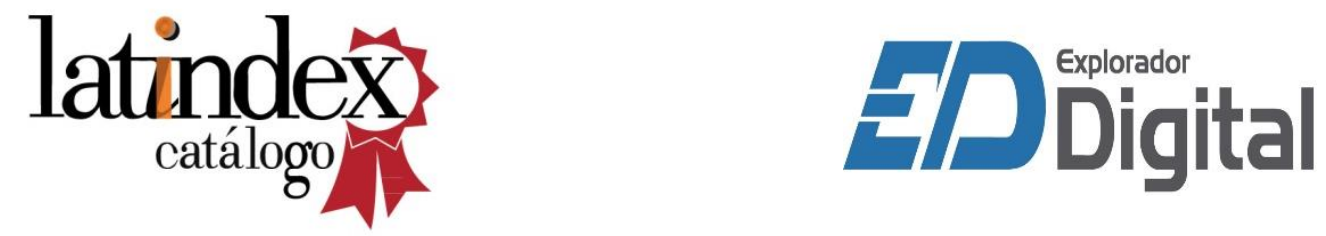